\title{
Human Motion Analysis in Treadle Pump Devices
}

\author{
C. Pereira', J. Malça' ${ }^{2}$, M. C. Gaspar ${ }^{3}$, and F. Ventura ${ }^{4}$ \\ ${ }^{1}$ Departamento de Engenharia Mecânica, \\ Instituto Superior de Engenharia de Coimbra, \\ Rua Pedro Nunes, 3030 - 199 Coimbra, Portugal. \\ candida@mail.isec.pt \\ ${ }^{2}$ Departamento de Engenharia Mecânica, \\ Instituto Superior de Engenharia de Coimbra, \\ Rua Pedro Nunes, 3030 - 199 Coimbra, Portugal. \\ jmalca@mail.isec.pt \\ ${ }^{3}$ Departamento de Engenharia Industrial, \\ Escola Superior de Tecnologia, Instituto Politécnico de Castelo Branco, \\ Av. do Empresário, 6000-767 Castelo Branco, Portugal. \\ calvete@est.ipcb.pt \\ ${ }^{4}$ Departamento de Engenharia Mecânica, \\ Faculdade de Ciências e Tecnologia da Universidade de Coimbra, \\ Pinhal de Marrocos, 3030-201 Coimbra, Portugal \\ fernando.ventura@dem.uc.pt
}

\begin{abstract}
Poverty and hunger are common problems in developing countries where agriculture is seriously affected by lacking of irrigated land. The treadle pump is an effective low cost device, which combines higher water discharge rates with ease of operation. Improving the performance of the treadle pump, considering dimensional and structural requirements, manufacturing and maintenance aspects, cost reduction and ergonomics is the aim of the authors. A human centered approach is proposed to enhance the performance of these pumps, firstly because the user's influence on the treadle pump's design has not been completely analyzed so far and secondly because water discharge depends significantly on the user's performance. A parametric study was carried out. It was found that a comfortable pumping position requires feet angular positions between $-10^{\circ}$ and $+10^{\circ}$ and treadles must be large enough to allow different pumping positions and operator's height. A new numerical approach is proposed for modeling the user's movement.
\end{abstract}

\section{Introduction}

Poverty associated to hunger are among the greatest problems in the world mainly in developing countries of Asia and Africa, where approximately one third of the population endure of chronic subnutrition. The agriculture is seriously affected in these regions where only $4 \%$ of arable land is irrigated. Thus, finding sufficient water for irrigation is one of the principle ways to increase production in agriculture and consequently one of the major challenges to improve food security and family incomes. In order to boost the production capacity of the typical farmer in Africa, lower-cost, water-efficient irrigation equipment is crucial. Among the most exciting innovations in manual irrigation technologies is the treadle pump $[1,2]$. The treadle

Please use the following format when citing this chapter:

Pereira, C., Malca, J., Gaspar, M. C., Ventura, F., 2006, in IFIP International Federation for Information Processing, Vo1ume 221, Human Work Interaction Design: Designing for Human Work, ed. T. Clemmensen, Campos, P., Omgreen, R., Petjersen, A1, and Wong, W., (Boston: Springer), pp. 135-145. 
pump is an effective low cost alternative to the rope and bucket system as it combines higher water discharge rates with ease of operation. Several beneficial impacts arise from the adoption of the treadle pump: $i$ ) improvement of family nutrition; ii) benefits on farming practices - increased agricultural area under irrigation, additional and new crops grown each season, reduced irrigation time, less tiring irrigation work - and iii) benefits at the economic level - high family incomes due to marketing of vegetables and employment opportunities for the whole supply chain of manufacturers, retailers and selling agents of treadle pumps. In this way, the treadle pump puts irrigation at the service of the poor.

A treadle pump comprises twin cylinders fitted with pistons that rise and fall when an operator, standing on the treadles connected to the pistons shifts his body weight side to side in a walking motion. In this manner, a suction pressure is created inside the cylinders and water is forced to enter the pump cylinders through the inlet pipe connected to the junction box. A non-return valve is fitted to the end of this pipe, allowing water to enter the pipe and preventing it from flowing back to the water source. Several designs of this device are currently available, all having the common basic components [3] as presented in figure 1 .

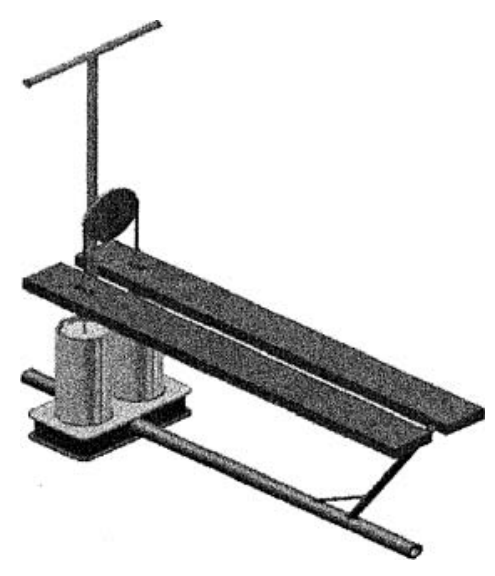

Fig. 31. The treadle pump

Different studies were developed comparing the performance of the treadle pump vis-à-vis other water-lifting options [4,5]. These studies showed that the treadle pump is easier to operate than other types of manual irrigation systems and its efficiency is also greater. Compared to other manual lifting devices, treadle pumps use human power in a relatively efficient way [6]. The pump employs the user's body weight and leg muscles, being much less tiring than other manual pumps that use the upper body and arm muscles [7]. Changing the driving power from arms and hands to feet and legs has been one of the most important innovations brought by the treadle pump.

The principles of human powered pumping systems depend mainly on the binomial operator-pumping device and must be completely understood to provide 
practical constructive solutions in order to enhance their performance. Thus, many features must be studied and enhanced so a multidisciplinary methodology focusing dimensional and structural requirements, manufacturing and maintenance aspects, cost reduction as well as ergonomic aspects is expected to obtain significant results in the treadle pump enhancement. This methodology is summarized in figure 2.



Fig. 32. Treadle pump enhancement methodology: key aspects

In this work, a human centered approach (user related issues) is proposed by the authors to enhance the performance of these manual pumping devices, firstly because the influence of the user on the treadle pump's design and constructive solutions has not been completely analyzed so far and secondly because the water amount pumped on this type of device depends significantly on the user and its performance. Hence, aspects like endurance, comfort and efficiency must be studied when considering the treadle pump's enhancement. The analysis of the treadle pump's ergonomic aspects is expected to help deriving new effective design directives. The gait and posture of the treadle pump's user, and in special the feet position, play a significant role on the performance of these human powered water lifting devices. In addition, the user's weight and physical shape are also essential, as the treadle pump's efficiency depends mainly on its operator's performance. Due to its operating simplicity, this device allows users like children and women $[3,6,7,8]$. Hence, the user's anthropometric influence must be carefully analyzed, which is the aim of the forthcoming section. 


\section{General considerations}

Persons using treadle pumps intend to elevate the maximum water volume within the shortest time. The pump and its mechanism must be efficient, however they are moved by human power and, therefore, persons are fundamental. Pumps must be actuated for long periods, so comfort of its user is essential, otherwise he will get tired rapidly. Besides, pumps can be used by males, females or children. Figure 3 illustrates

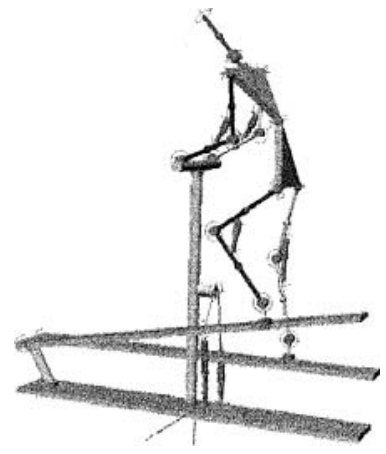

Fig. 33. Operator's position on the treadle pump

the posture of a man on the treadle pump. Ergonomics is the science that promotes adaptation of human and material resources for the best performance. This requires a deep knowledge of the human body in terms of anthropometry and comfort. Anthropometry is the discipline of design that studies human body sizes and it is strongly connected to ergonomy. The main aspects of the interaction between ergonomics and anthropometry are the sizes of human body segments, muscular forces, posture, and movements, since they are intimately related with comfort, safety and functionality.

Data on human body sizes vary from person to person and from country to country. They also vary from youth to eldness and with historical time. Height of Portuguese people has increased since antiguity and particularly during the XX century as a result of improvements in food and life conditions. Modern societies have recognized the importance of anthropometry and technical data is already available. However, in developing countries this is not a central issue and data is scarse.

The human body can be considered as a group of rigid segments (the bones) moved by muscles. Muscular fatigue is the decay in the capacity to generate muscular tension and occurs due to a sub-critical extended activity, or due to a high intensity and low duration activity. In a sub-critical exercise fatigue is a consequence of reduction in glycogen stocked in the muscles, even if oxygen is available. During prolonged exercise water ingestion and feeding are also fundamental. The fatigue of the human body is quickly reached when an isolate number of muscles is used or when the body is forced to work in an uncomfortable anatomic position. 


\section{Ergonomics of the Treadle Pump}

The performance of the treadle pump depends on the interaction of the triad operator-pump-mechanism as illustrated in figure 4.

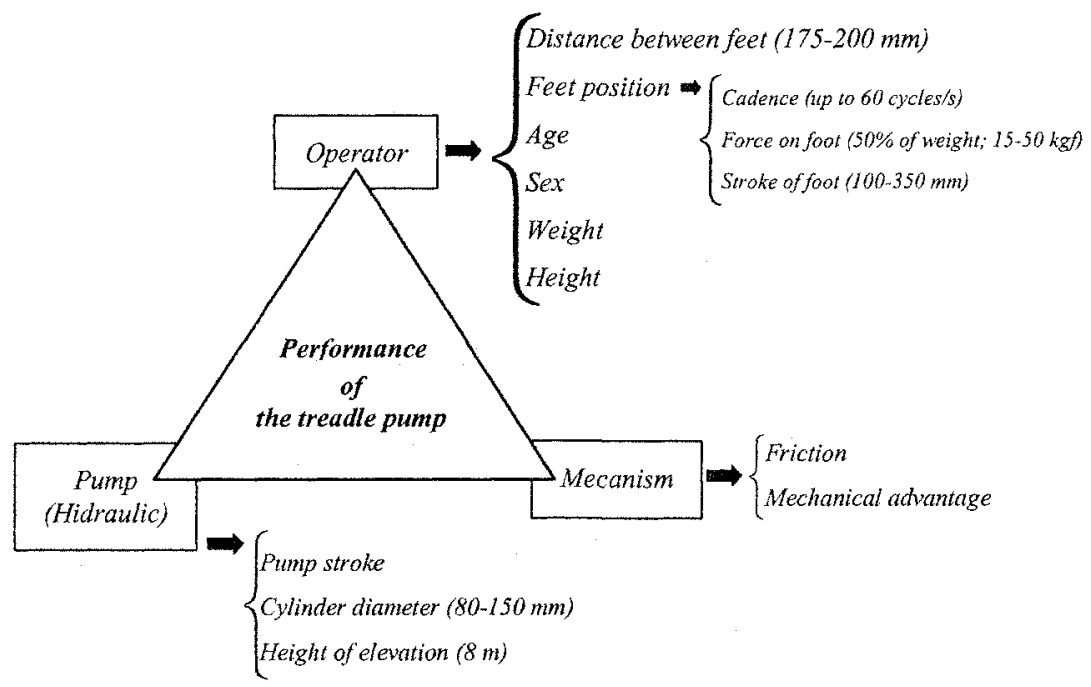

Fig. 34. Parameters determining the treadle pump performance

As referred above, ergonomic aspects are crucial when the aim is to improve treadle pump performance. Therefore, geometry and size of the different pump components should be designed and selected to guarantee the operator comfort and thus optimize the human power during large operating times. Unfortunately, few works can be found in the literature concerning this key aspect of the treadle pump. In 1993, Thomas [8] presented a definition of ergonomic efficiency as the relation between the (real) energy effectively transmitted by the operator to the treadles and the energy transmitted in ideal operation conditions. However, the parameters involved in this relation are difficult to quantify. Srinivas and Jalajakshi [5] evaluated the operacionality and ergonomic behavior of these devices inquiring several users. The results showed that the majority of the pump operators did not feel physical tiring in excess; nevertheless, the operating times were short. In fact, ergonomics is one dimension of the investigation where improvements are promising [3] and needed.

The treadle pump as a human powered device uses the operator's body weight and employs the most powerful muscles of the human body -leg muscles-instead of power from arms and hands to lift water from different sources. However, the use of these devices for long periods -up to 8 hours a day-requires physical strength, thus 
confort is crucial. So, in order to perform the optimization of this type of devices the first step is to study the issues related with the user.

The operator can be a male, female or child, as referred above, hence the parameters related with the operator can vary significantly, namely weight, height and physical shape. The treadle is a flexible machine allowing easy adaptation of different person, even children. The operator can select the foot position in relation to the pivot point (see figure 5), which modifies the cadence and the force on foot. Lower cadence corresponds to a higher force and lower stroke, which is adequate for heavier persons. On the other hand higher cadence, higher stroke and lower force are adequate to young people. Recommendations can be found in the literature for cadences lower than $60 \mathrm{cycles} / \mathrm{min}$ and foot strokes between $100-350 \mathrm{~mm}[3,8]$.

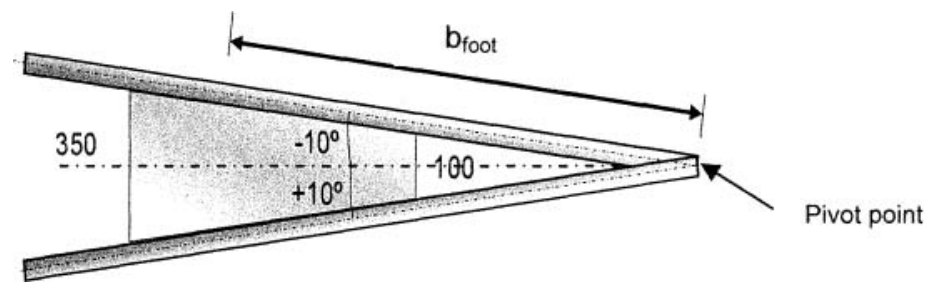

Fig. 35. Feet extreme positions related to the pivot point of treadles

The leg's movement is similar to the combination of step climbing and walking, so it is easily acquired and repeated sequentially in a mechanized way. However, the fatigue of human body is quickly reached when an isolate number of muscles are used or when the body is forced to work in an uncomfortable anatomic position. Furthermore, and for long periods, the force transmitted by the user to the treadle must not exceed $50 \%$ of his weight. For short periods this limit can be increased up to $70 \%$. Since the treadle pump can be used by different types of operators (males, females and children) it should be designed for resistance forces between 15 and 50 $\operatorname{kgf}[3,8]$.

The gait and posture of the treadle pump's user, and particularly the feet position, play a significant role on the efficiency of pumping. Figure 6 represents the extreme angular positions of feet. The equipment should be designed in order to guarantee that the mean position of operation matches a neutral angle of $\theta=0^{\circ}$-horizontal position- since in this position the foot force is maximum because the force acts vertically [9]. However, a comfortable pumping posture requires feet angular positions between $-10^{\circ}$ and $+10^{\circ}$. These values depend on the cylinder's position along the lever and on the cylinder's stroke [10]. Considering that the foot stroke should vary from 100 to $350 \mathrm{~mm}$ and $\theta$ between $-10^{\circ} \mathrm{a}+10^{\circ}$, as illustrated in figure 5 , then the distance from foot to the pivot point $-b_{\text {foof }}$ can vary between 280 and $1000 \mathrm{~mm}$. 


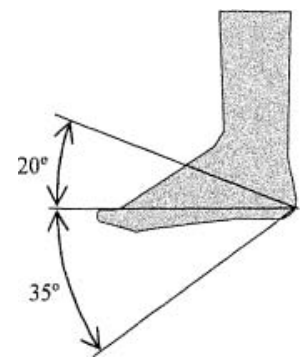

Fig. 36. Extreme angular positions of feet

The angular position of the legs depends on feet distance and represents another important ergonomic parameter (figure 7). In fact, treadles must be large enough to be suitable for different operator's height and allow selection of the best position. Additionally, relatively large treadles allow flexibility for the angular position of feet (see figure 8). On the other hand, enlargement of feet supports increases weight, with a negative impact in terms of pump's portability, which is another key design parameter.

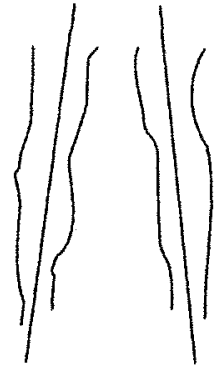

Fig. 37. Legs angular positions

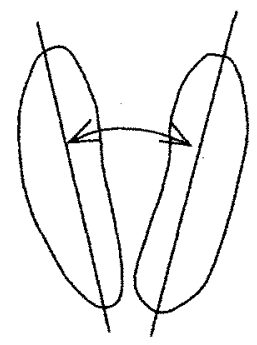

Fig. 38. Feet angular positions

\section{User's movement modelling:'The puppeteer model"}

As a future work, authors propose the development of numerical and experimental procedures to study the human body movement in a treadle pump. The interest in the analysis and simulation of different human actions stems from the need to predict with sufficient accuracy the mechanical behavior of the human body in various conditions of activity [10]. Human tracking plays an important role in this movement analysis, as it intends to recover continuously the global positions of the human body when in motion. The information thereby acquired can be used in many fields, such as virtual reality $[11,12]$, effect of industrial environments and activities [13,14], medicine (clinical study on orthopedic patients and rehabilitation) [15,16], sports (sport performance analysis and athletic training) $[17,18]$ or design of ergonomic 
equipment. Numerical studies of human body movement involve two main tasks: reconstructing the body and moving it. This is described in the next section.

\subsection{Body Modelling}

Different segments are usually considered, each representing a portion of the human body. A variable number of segments can be found in the literature. Ayoub et al. [13] have considered 5 segments in a 2D analysis. Ning et al. [19] have considered 14 rigid bodies -upper torso, lower torso, neck, two upper legs, two lower arms, two thighs, two legs, two feet and a head--, which are made of bone and soft tissues. Bone segments are considered non-deformable and therefore are represented as rigid bodies. Soft tissues may or may not be considered deformable. This last option is widely accepted in the literature, being the whole body segments regarded as rigid bodies. Tissue deformation may have inertial effects on movement kinetics during highly accelerated movements. Different anthropometric data is needed to reconstruct the body, namely distances, masses and inertia moments. This data varies significantly from person to person, which makes difficult its definition. Figure 9.a presents the human biomechanical model considered in the present work, based on the model proposed by Silva et al [10].

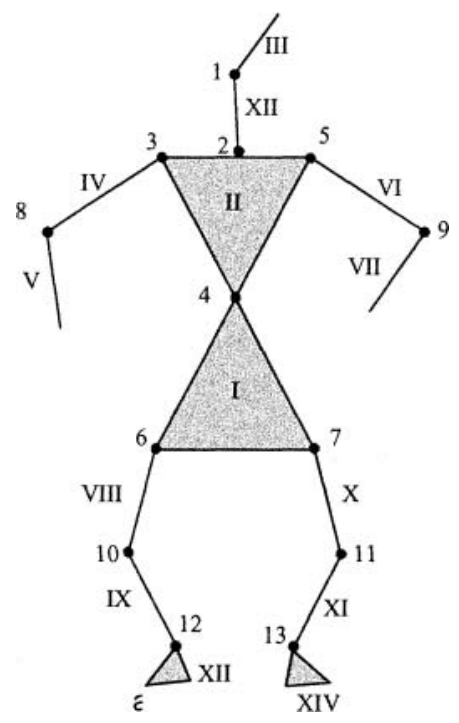

\begin{tabular}{|c|c|c|}
\hline Body no. & $\begin{array}{c}50 \% \text { Human Male } \\
L_{i}[\mathrm{~m}]\end{array}$ & $\begin{array}{c}50 \% \text { Human Male } \\
\text { Mass }[\mathrm{Kg}]^{*}\end{array}$ \\
\hline$I$ & 0.260 & 14.2 \\
\hline$I I$ & 0.250 & 24.9 \\
\hline HII & 0.230 & 4.24 \\
\hline$I^{r}$ & 0.320 & 1.99 \\
\hline$V$ & 0.260 & 1.84 \\
\hline$n$ & 0.320 & 1.99 \\
\hline$V I I$ & 0.260 & 1.84 \\
\hline$V I I I$ & 0.410 & 9.84 \\
\hline$D X$ & 0.385 & 4.81 \\
\hline$x$ & 0.410 & 9.84 \\
\hline$x y$ & 0.385 & 4.81 \\
\hline$X I I$ & 0.160 & 1.06 \\
\hline$x r I I$ & 0.053 & $1.62^{* * *}$ \\
\hline$X I y$ & 0.053 & $1.62 * *$ \\
\hline$L_{3 x+5}(a)$ & 0.375 & - \\
\hline$L_{6,7}(a)$ & 0.188 & - \\
\hline$L_{1-2}(b)$ & 0.199 & - \\
\hline$L_{l-3}(b)$ & 0.155 & - \\
\hline
\end{tabular}

Fig. 39. a) Human biomechanical model; b) Mass and dimensions of rigid bodies (*according to Silva et al. [10]; **- estimated values)

It has 14 rigid segments ( 2 for the head, 2 for the trunk and 10 for the members) and 13 kinematic joints. The total number of Cartesian coordinates is therefore $\mathrm{n}=14 \times 6=84$. Bodies IV-XI were assumed to have no rotation about their longitudinal 
axis ( 8 constraints). Joints 8,9 (elbows), 10,11 (knees) were assumed to be revolute joints ( $4 \times 5=20$ constraints). Joints $1,2,3,4,5,6,7,12,13$, were assumed to be spherical $(9 \times 3=27$ constraints). The mobility of the body is therefore $k=n-m=84-$ $55=29$ degrees of freedom. The data needed to implement the model comprises the mass, dimension, principal moments of inertia and center of mass location for each body. The table presented in figure $9 . \mathrm{b}$ includes mass and dimension data considered for each body segment. These parameters were used in motion computer modeling, using commercially available rigid multibody software. Figure 10 shows the biomechanical model as modeled in MSC.visualNastran.

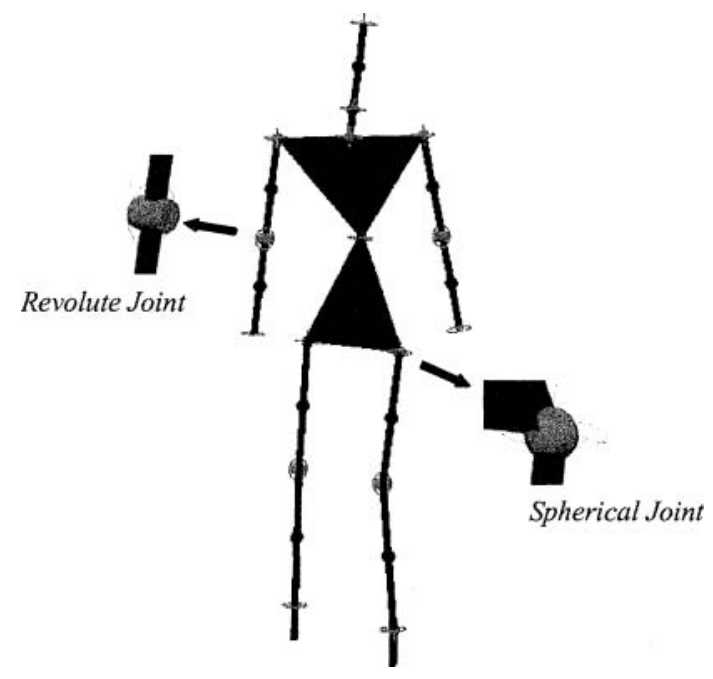

Fig. 40. Biomechanical model obtained using MSC.visualNastran software

\subsection{Body Movement}

Stereophotogrammetry methods are used to reconstruct 3-D landmark coordinates from photographs, radiographs and video images. Video based optoelectric systems are nowadays the most popular in movement analysis. These systems are used to track, by means of cameras, the 3-D position of a set of points. This allows a noninvasive estimation of the instantaneous position of points in a $3 \mathrm{D}$ measurement volume. Based in this concept, an experimental setup is being developed to track the movement of the operator in a treadle pump. Instantaneous positions of markers will be determined from a collection of digital images obtained by video cameras.

The rigid bodies that integrate the biomechanical model will be controlled (moved) by external actuators displacement/length type (also implemented in MSC.visualNastran software), instead of muscles, moving strategically the marker 
points of the model according to the previously tracked positions. Considering the similarity of this approach with the puppeteer control of a moved dummy, this procedure is named puppeteer model. The length of each actuator in each instant is obtained from experimental data (for each actuator is of time $v s$. length). The biomechanical model associated with the treadle pump parametric model, illustrated in figure 11, can be used to study the movement of the centre of mass during each cycle and to calculate the joint reactions and the resulting forces at the user's feet, in order to improve the performance of the binomial treadle pump/user. This is the next step of this research work.
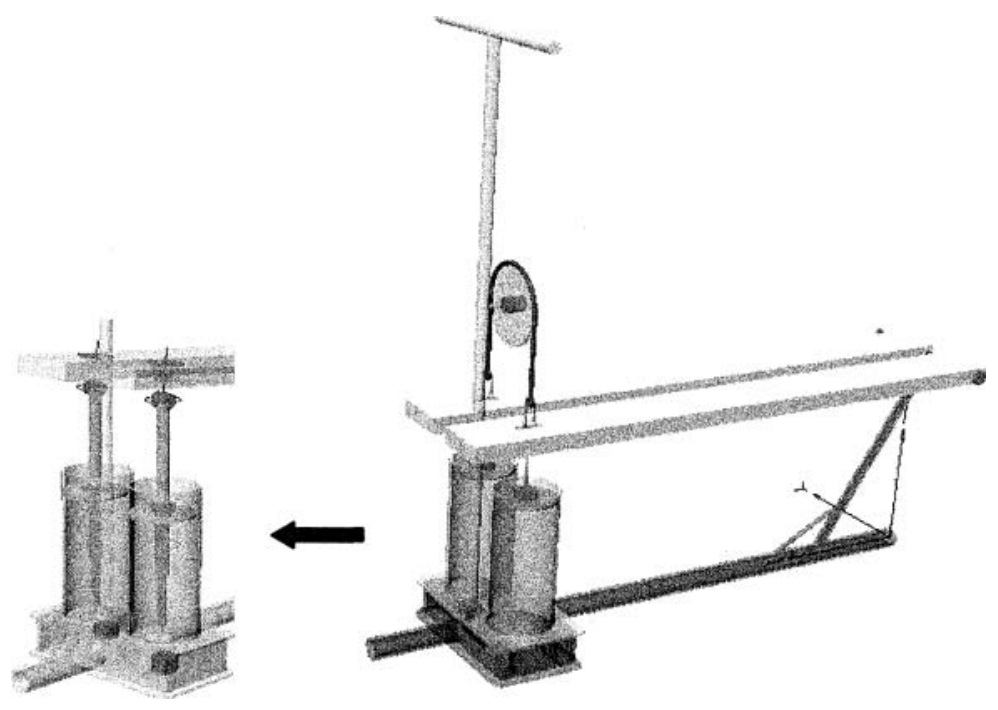

Fig. 41. Treadle pump modeled using MSC.visualNastran software

\section{Conclusions}

Improving the performance of the treadle pump, taking into account dimensional and structural requirements, manufacturing and maintenance aspects, cost reduction as well as ergonomic aspects is the aim of the present work. A human centered approach has been developed searching for improved comfort and thus optimized user's performance. The main parameters were identified and their limit values defined. Comfortable pumping position requires feet angular positions between $-10^{\circ}$ and $+10^{\circ}$. Treadles must be large enough to be suitable for different operator's height and to allow selection of the optimum position. Additionally, relatively large treadles allow flexibility for the angular position of feet.

Authors have proposed the development of numerical and experimental procedures to study the human body movement in a treadle pump. A biomechanical 
model in association with a treadle pump parametric model can be used to study the movement of the mass centre during each cycle and calculate the joint reactions and the resulting forces at the user's feet. As a result, improvements in the global performance of the treadle pump can be achieved.

\section{References}

1. Daka A.: Development of a Technological Package for Sustainable Use of Dambos by Small-Scale Farmers, PhD Thesis, University of Pretoria, South Africa. (2001)

2. Molden D, Amarasinghe U, Hussain I.: Water for Rural Development. International Water Management Institute. (2001).

3. Kay M, Brabben T.: Food and Agriculture Organization of the United Nations. Treadle pumps for irrigation in Africa, synthesis report. Rome. (2000).

4. Thomas, T.H.: The Performance Testing of Treadle Pumps. Working Paper no. 39, DTU, Department of Engineering, University of Warwick, Coventry, UK. (1993).

5. Srinivas S, Jalajakshi C.: Altenatives to Micro-Irrigation: Evaluation of the Treadle Pump. Economic and Political Weekly, September 18, (2004) 4271-4275.

6. Chigerwe J, Manjengwa N, van der Zaag P, Zhakata W, Rockström J.: Low head drip irrigation kits and treadle pumps for smallholder farmers in Zimbabwe: a technical evaluation based on laboratory tests. Physics and Chemistry of the Earth, 29: (2004) 10491059.

7. Perry E.: Low-cost irrigation technologies for food security in sub-Saharan Africa. In: Proceedings of the Workshop "Irrigation Technology Transfer in Support of Food Security", Harare, Zimbabwe, 14-17 April. (1997).

8. DTU - Development Technology Unit, The Treadle Pump, Working Paper 34, Dept of Engineering, Univ. of Warwick, Coventry, UK, (1991).

9. Panero, J., Zelnik, M.: Dimensionamento humano para espaços interiores, Ed. Gustavo Gili, S.A., Barcelona, (2002).

10.Silva, M.P.T., Ambrósio, J.A.C., Pereira, M.S.: Biomechanical Model with Joint Resistance for Impact Simulation. Multiboby System Dynamics. Vol.1 (1997) 65-84.

11.Cappozzo, A., Croce, U.D., Leardini, A., Chiari, L.: Human movement analysis using stereophotogrammetry - Part 1: theoretical background. Gait \& Posture. Vol 21. (2004) 186-196.

12.Theobalt, C. Carranza, J., Magnor, M. A., Seidel, H.-P.: Combining 3D flow fields with silhouette-based human motion capture for immersive video. Graphical Models. Vol.66 (2004) 333-351.

13.Ayoub, M.M.: A 2-D Simulation Model for Lifting Activities. Computers ind. Engng. Vol.35, (1998), 619-622.

14. Hostens, I. Ramon, H.: Descriptive analysis of combine cabin vibrations and their effect on the human body. Journal of Sound and Vibration. Vol.266 (2003) 453-464.

15.Wu, G.: Age-related differences in body segmental movement during perturbed stance in humans. Clinical Biomechanics. Vol.13 (1998) 300-307.

16.Aggarwal, J. K., Cai, Q.: Human Motion Analysis: A Review. Computer Vision and Image Understanding. Vol.7. (1999) 428-440.

17.Luo, Y., Wu, T.-, Hwang,J.-N.: Object-based analysis and interpretation of human motion in sports video sequences by dynamic bayesian networks. Computer Vision and Image Understanding. Vol.92. (2003) 196-216.

18.Pers, J., Bon, M., Kova, S., Sibila, M., Dezman, B.: Observation and analysis of large-scale human motion. Human Movement Science. Vol.21. (2002) 295-311.

19.Ning, H., Tan, T., Wang, L., Hu, W.: Kinematics-based tracking of human walking in monocular video sequences. Image and Vision Computing. Vol.22. (2004) 429-441. 